\title{
Water Chemisorption and Reconstruction of the MgO Surface
}

\author{
K. Refson, R. A. Wogelius and D. G. Fraser \\ Department of Earth Sciences, Parks Road, Oxford OX1 3PR \\ M. C. Payne and M. H. Lee and V. Milman* \\ Cavendish Laboratory, University of Cambridge, Madingley Road, Cambridge CB3 OHE
}

(December 6, 1994)

\begin{abstract}
The observed reactivity of $\mathrm{MgO}$ with water is in apparent conflict with theoretical calculations which show that molecular dissociation does not occur on a perfect (001) surface. We have performed ab-initio total energy calculations which show that a chemisorption reaction involving a reconstruction to form a (111) hydroxyl surface is strongly preferred with $\Delta E=-90.2 \mathrm{~kJ} \mathrm{~mol}^{-1}$. We conclude that protonation stabilizes the otherwise unstable (111) surface and that this, not the bare (001), is the most stable surface of $\mathrm{MgO}$ under ambient conditions.
\end{abstract}

PACS numbers: $82.65 \mathrm{My} 68.35 \mathrm{Md} 82.65 \mathrm{D} 71.10+\mathrm{x}$

Magnesium oxide has long provided a prototype for the study of surface structure and chemical reactions of oxides. Naturally occurring $\mathrm{MgO}$, known by its mineral name of periclase, is not a common crustal mineral, but its simple structure makes it an excellent example for the investigation of mineral surface chemistry.

Reactions at mineral surfaces are responsible for much of the chemical change which occurs in the Earth's crust. Weathering reactions control the erosion of rocks and the consequent evolution of surface topography thus providing an opposing mechanism to the more dramatic process of mountain building. Aqueous reactions in sedimentary basins are responsible for the diagenetic processes which transform unconsolidated sediments into rocks. In this work we study the nature of a simple mineral surface when exposed to an aqueous environment and the chemical interaction of water with that surface. This is both a prerequisite to studying the interaction with aqueous solutions and a tractable first step towards ligand-exchange reactions in more complex silicate minerals.

We have performed experiments on single-crystals of $\mathrm{MgO}$ prepared with high-quality (001) faces which were reacted with acidic solutions. The erperiments and results are reported in detail elsewheret, the main feature

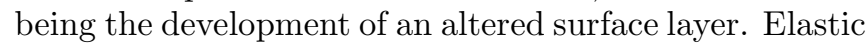
Recoil Detection Analysis (ERDA) shows protonation to a depth of $900 \AA$ with a $\mathrm{H} / \mathrm{Mg}$ ratio close to 2 giving a probable chemical composition of magnesium hydroxide. Indeed brucite (the mineralogical name for $\mathrm{Mg}(\mathrm{OH})_{2}$ ) is the most common alteration product of periclase in the natural environment 1 and well-crystallized intergrowths of brucite on periclase have been reported 5 .

The initial stage in the reaction is hydroxylation of the surface. $\mathrm{MgO}$ has the cubic rocksalt structure with (001) cleavage planes. This is the most stable surface and is the only one seen experimentally 6 . The simplest possibility for a hydroxylated surface is obtained by dissociating a water molecule and placing the $\mathrm{OH}$ group above each magnesium ion and the $\mathrm{H}$ above each oxygen of the (01) surface (see Fig. 17a) as postulated by Coluccia et al.

Some striking hydroxylation experiments were reported by Jones et al. who studied surface roughening on (001) faces of nanocfystalline $\mathrm{MgO}$ in a transmission electron microscope 8 . The remarkable affinity of $\mathrm{MgO}$ for water is demonstrated by their in situ observation of hydration-induced surface roughening over 10 minutes under vacuum with $\mathrm{P}_{\mathrm{H}_{2} \mathrm{O}}<10^{-5} \mathrm{~Pa}$. The presence of surface hydroxyl groups on $\mathrm{MgO}$ powders exposed to $\mathrm{H}_{\mathrm{f}} \mathrm{O}$ has been demonstrated by infra-red spectroscopy 0 . 10. Hydroxyls are clearly distinguishable from physisorbed molecular water by the $\mathrm{HOH}$ bending mode which disappears above $100^{\circ} \mathrm{C}$, while the $\mathrm{OH}$ stretching mode persists even above $500^{\circ} \mathrm{C}$. Furthermore there is complete monolayer coverage of the surface by hydroxyls, as shown by microgravimetry measurements.

Despite these observations, the most reliable theoretical calculations predict that water molecules do not dissociate on the (001) surface. Scamehorn et al.11 calculated the energetics of the reaction $\{>\mathrm{MgO}\}+\mathrm{H}_{2} \mathrm{O} \rightleftharpoons\left\{>\mathrm{Mg}(\mathrm{OH})_{2}\right\}$ to form the hydroxylated (001) surface using periodic Hartree-Fock methods. They showed that dissociative chemisorption is energetically unfavourable and that physisorption of intact water molecules is preferred. This was confirmed by a CarParrinello $a b$ initio molecular-dynamics study 12 which investigated the dynamics of a water molecule at a $\mathrm{MgO}$ (001) surface. No dissofiation occurred. Experiments performed by Jones et al. $\mathrm{B}$ also led to the conclusion that perfect (001) surface sites are not protonated.

In summary, water demonstrably chemisorbs onto $\mathrm{MgO}$ but trustworthy calculations show that $\mathrm{H}_{2} \mathrm{O}$ molecules should not dissociate on the only known stable surface.

Several authors have proposed that water dissociates

*Present address: Molecular Simulations, 240/250 The Quorum, Barnwell Road, Cambridge CB5 8RE, UK 

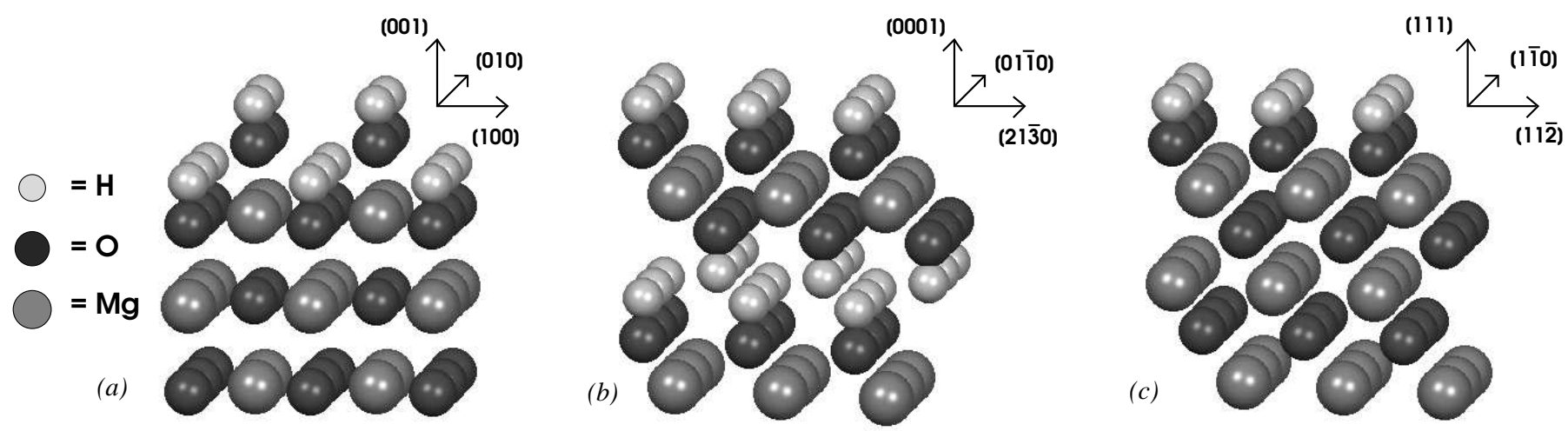

FIG. 1. (a) Hypothetical hydroxylated (001) surface of $\mathrm{MgO}$, (b) (0001) surface of $\mathrm{Mg}(\mathrm{OH})_{2}$, (c) our postulated (111) hydroxyl surface of $\mathrm{MgO}$. The top three $\mathrm{O}-\mathrm{Mg}-\mathrm{O}$ layers of an oxygen-terminated $\mathrm{MgO}$ (111) surface have the same structure as $\mathrm{Mg}(\mathrm{OH})_{2}$ (0001). The (111) hydroxyl surface may be equivalently constructed by protonation of an oxygen-terminated surface or by hydroxylation of a magnesium-terminated surface.

instead at low co-prdinated sites such as steps, corners and other defects 13 and dissociation yas demonstrated computationally at steps and corners 12.14 . However one might expect this to simply saturate the defect sites. Dissociation at defects does not explain the monolayer coverage of the surface by $\mathrm{OH}$ group the whole-surface roughening observed in the TEM nor the progressive transformation of the entire (001) surface and incipient bulk hydroxylation observed by i.r. spectroscopyte 6 and in our dissolution experiments.

In order to account for these phenomena we propose an alternative structure for the fully hydroxylated surface. The progressive formation of a bulk hydrated layer suggests consideration of the structural relationship between the oxide and the crystalline hydroxide. $\mathrm{Mg}(\mathrm{OH})_{2}$ is trigonal and is composed of layers of $\mathrm{Mg}^{2+}$ and $\mathrm{OH}^{-}$ions in (0001) planes (see Fig. 1 b). This "basal" plane is also the cleavage plane, yielding a stable type II hydroxide surface. The trigonal symmetry means that the basal plane is incommensurate with the cubic (001) $\mathrm{MgO}$ surface, but closely resembles the threefold symmetric (111). Fig. 11 illustrates that protonation of the (111) creates a surface with the same structure as the $\mathrm{Mg}(\mathrm{OH})_{2}$ (0001) cleavage plane. This strongly suggests that protonation may stabilize the $\mathrm{MgO}$ (111) surface, and that this may be the hydroxylated surface observed by i.r. spectroscopy.

Supporting evidence is provided by well-crystallized natural growths of $\mathrm{Mg}(\mathrm{OH})_{2}$ on $\mathrm{MgO}^{5}$ and from the inverse reaction, the dehydration of $\mathrm{Mg}(\mathrm{OH})_{2}$ to form $\mathrm{MgO}$. 5 . In both cases an epitaxial relationship exists between the two phases such that the [0001] axis of the hydroxide is aligned with with the oxide [111].

We have investigated the stability of the (111) hydrowyl surface using total-energy pseudopotential calculations 16 . The density functional theory formulation of quantum mechanics is solved within the local density approximation (using the parameterization of Perdew and Zunger 17 ) by conjugate-gradient minimisation of the total energy with respect to the valence electron wavefunctions. Optimized non-local pseudopotentials are used in the Kleinman-Bylander separable representation. The system of ions and electrons is subject to periodic bound- ary conditions which allows the use of a plane-wave basis set. The latter gives an accurate representation of the crystalline environment. Just as importantly, it permits analytic forces to be computed allowing for relaxation of the ions to their minimum energy configuration. This type of calculation has been used to study defect energies in $\mathrm{MgO}^{18}$, $\mathrm{OH}$ groups as substitutional defects in quartz 19 , reconstruction of the silicon (111) surface 20 and dissociation of $\mathrm{Cl}_{2}$ at a silicon surface 21 .

One difficulty in using plane-wave pseudopotential methods for oxides has been that the tightly-bound oxygen $2 p$ electrons require a high energy cutoff making the calculations expensive to perform. Recent advances have dramatically improved the convergence properties so that oxide calculations are now routine22. In this work we used a new optimized oxygen pseudopotential which gives complete convergence of the electronic energy (to $0.05 \mathrm{eV}$ from an energy of $909 \mathrm{eV}$ ) at a cutoff of $500 \mathrm{eV}$. Magnesium was also represented by an optimized pseudopotential and hydrogen by a pure coulombic potential.

As a check on the accuracy to be expected we performed calculations on bulk $\mathrm{MgO}$ and an isolated water molecule, the initial reactants. The results agree closely with experimental values and are summarised in table [.

\begin{tabular}{rrrr}
\hline \hline & Property & Calculated & Experimentä \\
\hline $\mathrm{MgO}$ & $\mathrm{a}_{0}(\AA)$ & $4.217 \pm 0.001$ & 4.211 \\
& $\mathrm{~K}(\mathrm{Mbar})$ & $1.62 \pm 0.02$ & $1.603 \pm 0.003$ \\
& $\mathrm{~K}^{\prime}$ & $4.19 \pm 0.08$ & $4.15 \pm 0.102$ \\
$\mathrm{H}_{2} \mathrm{O}$ & bond length $(\AA)$ & 0.966 & 0.9572 \\
& $\mathrm{HOH}$ angle $\left({ }^{\circ}\right)$ & $103.9-104.1^{\mathrm{a}}$ & 104.52 \\
\hline \hline
\end{tabular}

TABLE I. Calculated properties of bulk $\mathrm{MgO}$ and isolated water molecule: $\mathrm{MgO}$ lattice parameter $\mathrm{a}_{0}$, bulk modulus $\mathrm{K}$ and $K^{\prime}=d K / d P ; \mathrm{H}_{2} \mathrm{O}$ molecular bond length and angle.

${ }^{\text {a }}$ The value depends on the size and shape of the supercell and the orientation of the molecule, indicating a very small interaction between a molecule and its periodic images.

To represent a surface using periodic boundary conditions we performed calculations on a slab of $\mathrm{MgO}$ in a periodic cell of larger dimension leaving a vacuum sep- 
arating periodic images of the slab. The surface energy is given by $\Delta E_{\text {surf }}=\left(E_{\text {slab }}-E_{\text {bulk }}\right) / a^{2}$. To test convergence with respect both to the number of layers in the slab and the vacuum space we calculated the energy of the (001) $\mathrm{MgO}$ surface using 4, 6 or 8 atomic layers in the slab and different $c$ dimensions of the supercell leaving between 8 and $16 \AA$ of vacuum. The periodic cells had dimensions $a_{0} / \sqrt{2} \times a_{0} / \sqrt{2} \times n a_{0}, n=1,4,6$. The lattice parameter $a_{0}$ was fixed at the experimental value of $4.2117 \AA$. The $\mathrm{k}$ point set contained 8 points for the long cells and 32 or 48 points for the compact cell used for the bulk calculation. In every case relative energies were computed using equivalent sets to cancel basis-set size errors. The total energies were well-converged as a function of k-point sampling, to better than $0.05 \mathrm{eV}$ out of $909 \mathrm{eV}$. The co-ordinates of the ions in the outer layers of the slab were relaxed to their minimum energy configuration, while the inner 2 layers were constrained at their bulk separation. The surface $\mathrm{Mg}^{2+}$ ions moved inwards by $1 \%$ and the $\mathrm{O}^{2-}$ ions by $0.1 \%$ in agreement with previous Hartree-Fock calculations 23 . The resultant surface energies are all between 1.093 and $1.103 \mathrm{~J} \mathrm{~m}^{-2}$ which shows that a 4-layer slab with $8 \AA$ of vacuum gives energies converged to a precision of better than $0.01 \mathrm{~J} \mathrm{~m}^{-2}$. This result is compared with previous work in table II].

\begin{tabular}{lr}
\hline \hline Technique & $\Delta E_{\text {surf }}\left(\mathrm{J} \mathrm{m}^{-2}\right)$ \\
\hline DFT/LDA this work & 1.10 \\
DFT/LDA using Gaussian basis set 30 & 1.16 \\
Periodic HF & 1.43 \\
Pair Potentials $^{\mathrm{B} 1}$ & 1.07 \\
Experiment $^{\mathrm{b}}$ & $1.04-1.20$ \\
\hline \hline
\end{tabular}

TABLE II. Calculated $\mathrm{MgO}(001)$ surface energies and comparison with previous measurements and calculations.

a These calculation 23 used basis set containing only $s$ and $p$ orbitals. Birkenheuer et al 30 showed that including $d$ orbitals

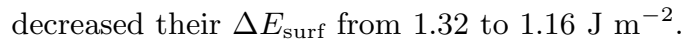

${ }^{\mathrm{b}}$ summarised by Tosi 32 , andrefstherein.

The test calculations establish that errors in the energy differences due to cell-size and basis-set effects are less than $1 \mathrm{~kJ} \mathrm{~mol}^{-1}$. This leaves one significant source of systematic error, the LDA, which is known to underestimate molecular dissociation energies 21. We estimated an upper bound for this error of $40 \mathrm{~kJ} \mathrm{~mol}^{-1}$ from a calculation of the reaction $\mathrm{MgO}+\mathrm{H}_{2} \mathrm{O} \rightleftharpoons \mathrm{Mg}(\mathrm{OH})_{2}$ (brucite) whose energy is well known . Our main result is quite robust to an error of this magnitude.

The chemisorption energy is fully determined given the structure and state of the reactants and products by $\Delta E=E_{\text {product }}-\sum E_{\text {reactants }}$. The appropriate initial states are the bare (001) surface of $\mathrm{MgO}$ and ice (since this is a zero-temperature calculation) and the final structures are the hydroxylated (001) and the (111) hydroxyl surfaces of Fig. 11. Scamehorn et al. showed that the fully hydroxylated (001) surface has lower energy than if partially hydrated 11 so we performed calculations for only that state. Calculations on the hydroxylated (001) surface were performed in a supercell $\sqrt{2} a_{0} \times \sqrt{2} a_{0} \times 4 a_{0}$ containing 4 layers of $\mathrm{Mg}$ and $\mathrm{O}$ ions plus 2 surface layers of hydrogen or hydroxyl. Simple electrostatic considerations indicate that full hydroxylation must also be favoured for the (111) surface: this is the only means of achieving a non-polar surface.

Ideally calculations on the reactants and products should use equivalent supercells to achieve cancellation of basis set errors in the computed $\Delta E$. This rules out a direct calculation for the bare $(001) \rightleftharpoons(111)$ hydroxyl reaction as the symmetries differ and no cell can accommodate both structures. However the reaction may be split into two stages $\{>\mathrm{MgO}\}^{(001)} \rightleftharpoons \mathrm{MgO}^{\text {bulk }}$ and $\mathrm{MgO}^{\text {bulk }}+\mathrm{H}_{2} \mathrm{O} \rightleftharpoons\left\{>\mathrm{Mg}(\mathrm{OH})_{2}\right\}^{(111)}$ whose partial energies sum to the desired result. Each stage can be computed using cells of the appropriate symmetry since bulk $\mathrm{MgO}$ is commensurate with both. The first stage is simply the surface energy. The second used trigonal cells with $a=a_{0} / \sqrt{2}, c=\sqrt{3} a_{0}$ and $24 \mathrm{k}$-points for bulk MgO and $a=a_{0} / \sqrt{2}, c=2 \sqrt{3} a_{0}$ with $12 \mathrm{k}$-points and contained 5 layers of $\mathrm{Mg}$ and $\mathrm{O}$ ions in a hydroxylated slab. The outer layers were relaxed in all these calculations.

The initial state of water was based on calculations of an isolated molecule in supercells equivalent to those used for the bulk $\mathrm{MgO}$ calculations but of twice the linear dimensions to minimize interaction between periodic images. To this we added the experimental value for the sublimation energy of ice, $-47.35 \pm 0.02 \mathrm{~kJ} \mathrm{~mol}^{-124}$.

The results are listed in table III. As expected the hydroxylation of the (001) surface is energetically unfavourable. Our hypothesis that hydroxylation stabilizes the (111) surface is confirmed, with a $\Delta E$ of $90.2 \mathrm{~kJ} \mathrm{~mol}^{-1}$ with respect to ice, or (by using the results of Scamehorn et al 11 for the physisorption energy) $-117.3 \mathrm{~kJ} \mathrm{~mol}^{-1}$ relative to physisorbed water. This provides a ready explanation of the spectroscopic observations of surface hydroxyls and the observed reactivity of $\mathrm{MgO}$ with water manifested as rapid roughening of the (001) surfaces of microcrystals. The chemisorption reaction must involve a reconstruction of the (001) surface.

\begin{tabular}{lcrr}
\hline \hline Initial & Final & $\Delta E\left(\mathrm{~J} \mathrm{~m}^{-2}\right)$ & $\Delta E\left(\mathrm{~kJ} \mathrm{~mol}^{-1}\right)$ \\
\hline$(001)$ & $(001)$ & +0.78 & +41.6 \\
bulk & $(111)$ & -0.59 & -31.3 \\
$(001)$ & $(111)$ & -1.69 & -90.2 \\
\hline \hline
\end{tabular}

TABLE III. Computed energies for the chemisorption reaction of water with $\mathrm{MgO}$, formally $\{>\mathrm{MgO}\}^{\text {initial }}+\mathrm{H}_{2} \mathrm{O}$ $\rightleftharpoons\left\{>\mathrm{Mg}(\mathrm{OH})_{2}\right\}^{\text {final }}$. The initial states are bulk $\mathrm{MgO}$ or the (001) surface plus ice at $0 \mathrm{~K}$. The final states are the hydroxylated (001) or (111) hydroxyl surfaces. The (111) surface energies are expressed per unit of the original (001) surface area assuming a ratio of $\sqrt{3}: 1$. A periodic HF study found a significantly more positive $\Delta E$ for the hydroxylation of (001) of 77-90 kJ mol $\mathrm{kJ}^{-11}$. The measured enthalpy of water chemisorption on $\mathrm{M}_{\mathscr{\phi}} \mathrm{q}$ at $613 \mathrm{~Pa}$ and $543 \mathrm{~K}$ is in the range -113 to $-189 \mathrm{~kJ} \mathrm{~mol}^{-133}$. Approximate thermodynamic corrections to $\Delta E$ at $0 \mathrm{~K}$ for the (111) hydroxyl give $\Delta H_{543} \approx$ $-138 \mathrm{~kJ} \mathrm{~mol}^{-1}$, consistent with those experiments.

We conclude that the (111) hydroxyl, rather than the 
bare (001) is the normal surface of $\mathrm{MgO}$ under ambient environmental conditions. A (001) MgO surface will chemisorb water and reconstruct except under ultra-high vacuum or high temperature. Dehydroxylation is experimentally observed only under UHV 13 : indeed hydration reactions occur under lesser vacuum in the TEM at a partial pressure of water of $<10^{-5} \mathrm{~Pa}$. Upon heating dehydroxylation begins at $200^{\circ} \mathrm{C}$ but is gradual with residual hydroxyls persisting until over $700^{\circ} \mathrm{C} / 10$.

This solves a number of experimental puzzles. 1) The $\mathrm{H}_{2} \rightleftharpoons \mathrm{D}_{2}$ exchange reaction is catalysed by $\mathrm{MgO}$ at temperatures as low as $78 \mathrm{~K} 25$. Structural surface protons provide the necessary exchange site26. 2) It may also resolve discrepancies between experimental and theoretical energies of adsorption of $\mathrm{CO}$ onto $\mathrm{MgO}_{27}$. The appropriate surface for molecular adsorption is the (111) hydroxyl surface, not the bare (001). 3) Vermilyea showed that the dissolution rates of $\mathrm{MgO}$ and $\mathrm{Mg}(\mathrm{OH})_{2}$ are identical over the $\mathrm{pH}$ range $2-528$, an observation easily explained by the almost identical surface structures.

Identification of the actual reconstruction pathway is beyond the scope of this paper, but a plausible mechanism must account for both the dissociation of the water molecule and the transport of surface $\mathrm{Mg}^{2+}$ and $\mathrm{O}^{2-}$ ions. Previous $a b$ initio calculations have shown that water molecules dissociate at surface defects, particularly steps and corners 1214 , corroborating the observation that the reconstruction is more rapid if the surface is damaged 3 . The activation barrier for ion transport may be estimated given that the inverse reconstruction, the annealing of $\{100\}$ facets on a (111) surface, is observed to occur at $1000 \mathrm{~K} 13$. The equivalent thermal energy is $12 \mathrm{~kJ} \mathrm{~mol}^{-1}$, rather less than the chemical energies of the hydroxylation reaction. It is also possible that the barrier for $\mathrm{Mg}^{2+}$ transport away from the defect site is reduced by hydration with either the product $\mathrm{OH}^{-}$ion or an intact $\mathrm{H}_{2} \mathrm{O}$ molecule. This would expose low coordinated oxygen sites able to dissociate incoming water molecules and thereby continue the process.

Stabilization of a type III polar surface by protonation or hydroxylation to form a non-polar type II surface is unlikely to be unique to $\mathrm{MgO}$. We would also expect reconstructive chemisorption to occur in other fcc binary metal oxides such as $\mathrm{NiO}$.

The consequences of the stability of the hydroxyl surface over the bulk are substantial but rather harder to predict. It does provide a driving force for the layer hydration observed by us and others which apparently leads to a topotactic oxide-hydroxide transformation. However a much better characterization of the hydroxylated layer is needed and further studies to establish its composition and structure are under way. Whatever its nature it is demonstrably a vital precursor stage in the dissolution of magnesium oxide and may also prove relevant to understanding recrystallization and precipitation reactions.

Acknowledgment: This work was supported by NERC under grants GR3/8970 and GR3/8310.
${ }^{1}$ R. A. Wogelius et al., Geochim. Cosmochim. Acta (in press).

${ }^{2}$ W. H. Casey, H. R. Westrich, and G. W. Arnold, Geochim. Cosmochim. Acta 53, 2795 (1988).

${ }^{3}$ The braces are used to denote a surface species and the $>$ symbol indicates the bond to the substrate.

${ }^{4}$ W. A. Deer, R. A. Howie, and J. Zussman, An Introduction to the Rock Forming Minerals (Longman, Harlow, Essex CM20 2JE, UK, 1966).

${ }^{5}$ A. I. Gorshkov, Y. V. Kolodyazhnaya, A. V. Mohkov, and P. P. Smolin, Doklady Rossiyskoy Akademii Nauk 322, 584 (1992).

${ }^{6}$ E. A. Colbourn, Surface Science Reports 15, 281 (1992).

${ }^{7}$ S. Coluccia, L. Marchese, S. Lavagnino, and M. Anpo, Spectrochim. Acta A 43, 1573 (1987).

${ }^{8}$ C. F. Jones et al., J. Chem. Soc. Faraday Trans. 1 80, 2609 (1984).

${ }^{9}$ Y. Kuroda et al., J. Chem. Soc. Faraday Trans. 1 84, 2421 (1988).

${ }^{10}$ E. Knozinger, K. H. Jacob, S. Singh, and P. Hofmann, Surf. Sci. 290, 388 (1993).

${ }^{11}$ C. A. Scamehorn, A. C. Hess, and M. I. McCarthy, J. Chem. Phys. 99, 2786 (1993).

${ }^{12}$ W. Langel and M. Parrinello, Phys. Rev. Lett. 73, 504 (1994).

${ }^{13}$ H. Onishi, C. Egawa, and T. Aruga, Surf. Sci. 191, 479 (1987).

${ }^{14}$ C. A. Scamehorn, N. M. Harrison, and M. I. McCarthy, J. Chem. Phys. 101, 1547 (1994).

15 J. F. Goodman, Proc. Roy. Soc. A247, 346 (1958).

${ }^{16}$ M. C. Payne et al., Rev. Mod. Phys. 64, 1045 (1992).

17 J. Perdew and A. Zunger, Phys. Rev. B 23, 5048 (1981).

18 A. De Vita et al., Phys. Rev. Lett. 68, 3319 (1992).

19 J. S. Lin, M. C. Payne, V. Heine, and J. D. C. McConnell, Phys. Chem. Min. 21, 150 (1994).

${ }^{20}$ I. Stich, M. C. Payne, R. D. King-Smith, and J. S. Lin, Phys. Rev. Lett. 68, 1351 (1992).

${ }^{21}$ A. De Vita et al., Phys. Rev. Lett. 71, 1276 (1993).

22 J. S. Lin, A. Qteish, M. C. Payne, and V. Heine, Phys. Rev. B 47, 4174 (1993).

${ }^{23}$ M. Causa, R. Dovesi, C. Pisani, and C. Roetti, Surf. Sci. 175, 551 (1986).

${ }^{24}$ D. S. Eisenberg and W. Kauzmann, The Structure and Properties of Water (OUP, Oxford, 1969).

${ }^{25}$ M. Boudart et al., J. Am. Chem. Soc. 94, 6622 (1972).

${ }^{26}$ A. B. Kunz and M. P. Guse, Chem. Phys. Lett. 45, 18 (1977).

${ }^{27}$ M. A. Nygren, L. G. M. Pettersson, Z. Barandiaran, and L. Seljo, J. Chem. Phys. 100, 2010 (1994).

${ }^{28}$ D. A. Vermilyea, J. Electrochem. Soc. 116, 1179 (1969).

${ }^{29}$ I. Jackson and H. Niesler, in High Pressure Research in Geophysics, edited by S. Akimoto and M. H. Manghnani (Center for Academic Publishing, Tokyo, 1982), p. 93.

${ }^{30}$ U. Birkenheuer, J. C. Boettger, and N. Rosch, J. Chem. Phys. 100, 6826 (1994).

${ }^{31}$ W. C. Mackrodt, Phys. Chem. Min. 15, 228 (1988).

${ }^{32}$ M. P. Tosi, Solid State Phys. 16, 1 (1964).

${ }^{33}$ D. Beruto, A. W. Searcy, R. Botter, and M. Giordani, J. Phys. Chem. 97, 9201 (1993). 